\title{
Justification of the basic algorithms of the mine safety information system
}

\author{
Volodymyr Shevchenko ${ }^{1}$, and Anton Slashchov ${ }^{1, *}$ \\ ${ }^{1}$ Institute of Geotechnical Mechanics named by N. Poljakov of National Academy of Sciences of \\ Ukraine, 49005, Dnipro, Simferopolska Str., 2a, Ukraine
}

\begin{abstract}
This article is devoted to substantiation of basic algorithms for the information system which could provide prompt making of decisions on ensuring safety of underground mining jobs, which are of great importance for the job safety at the mining enterprises. The information safety system architecture and some basic algorithms was developed. The system differs by its methods for prompt predicting and assessing of different scenarios of geomechanical process development, and which includes the following subsystems: a basic client-server subsystem with functions of interaction between the personnel and management of the enterprise; a reference and information subsystem, which supports a decision making process, accumulates data and analyzes technical documentation; a subsystem for analyzing the job safety by geomechanical factors and for assessing of the "support-rocks" system state basing on the risk criteria and mathematical fuzzy logics. Two integral indicators of safety are formed. The first indicator is used to control entering of the control object to the emergency mode and to determine a factor, which requires urgent interruption, and the second indicator is used for the total assessment of the object current state.
\end{abstract}

\section{Introduction}

In Ukrainian coal mines, rate of the face advance is increased, the mines are transferring to the roof bolting and pillar-free technology for supporting the roadways in order to reuse them in future, and all these factors are realized in extremely dangerous conditions. High danger of the coal and other mines is associated with objective geomechanical factors, as the minerals are extracted from the weak and water-saturated rocks at the great depths. Under such conditions, uncontrolled deformation of the rock mass is occurred in the form of sudden rock fall, destruction of the roof supports and blockage of the roadways, accompanied with the traumatism of up to half of the total number of the casualties. Besides, analysis of accidents shows that they are less commonly caused by equipment failure: human factor ranks the first. Therefore, an effective way to reduce accidents and injuries is to predict the geomechanical processes and strictly control observance of the safety rules by personnel with the help of the latest information systems [1-3]. Therefore,

\footnotetext{
*Corresponding author: AISlashchov@nas.gov.ua
} 
the justification of the basic algorithms of the information system which could provide prompt making of decisions on ensuring safety of underground mining jobs - is the live scientific task, which is of great importance for the job safety in the mining enterprises.

\section{Methods}

The methodology for building the information system for mine safety should include methods of operational management of personnel and estimating different scenarios of the geomechanical processes development. The information safety system (ISS) architecture designed as the three main subsystems. The first is a basic client-server subsystem, which, with the help of network technologies and mobile communication, provides functions of interaction between the personnel and management of the enterprise. The second is a reference and information subsystem, which supports a decision-making process through the data accumulation and storage, information presentation as requested and automatic analysis of needed normative and technical documentation. The third subsystem assesses rate of the job safety by geomechanical factors and with the help of mathematical models. This is a system for supporting the decision-making with elements of expert knowledge, which accumulates and analyzes knowledge (for example, typical mining and geological conditions or standard scenarios of the rock massive behavior) and has algorithms for assessing state of the "support-rocks" system on the basis of danger criteria and mathematical fuzzy logics.

\section{Results and discussion}

Organization of personnel management assumes that the text description of the tasks, time for their accomplishment, explanatory images, additional requirements and instructions and on-line information concerning monitoring of the current task accomplishment are transmitted to and recorded by the electronic media. The subsystem of personal management has a warning function for rapid response to the occurrence and development of events caused, in the first turn, by geomechanical factors, Figure 1. This function helps to make appropriate decisions during essentially shorter period of time. Labor safety is improved thanks to better interaction between the people and their more strict disciplinary responsibility.

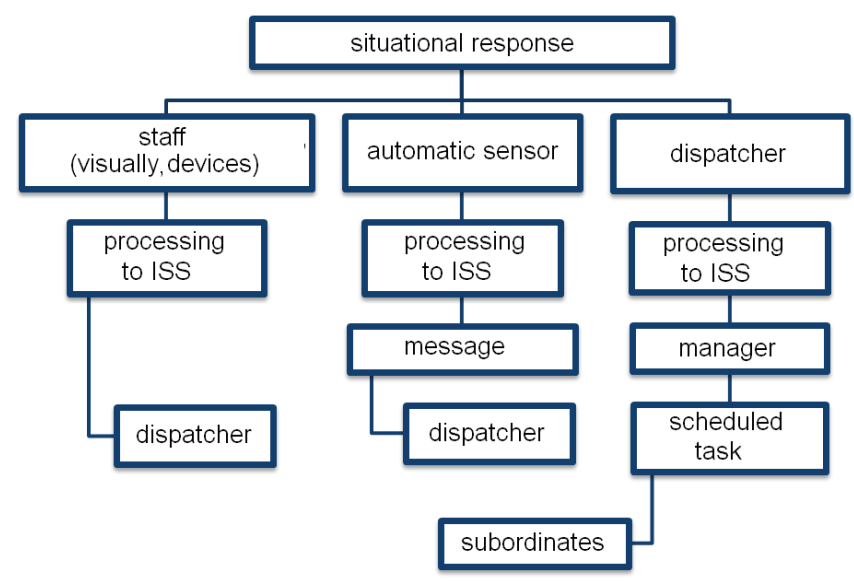

Fig. 1. Responsiveness of the personnel management system to the changed production situation.

A number of basic algorithms have been developed for the personnel management 
information system, which are designed with the possibility of rapidly increasing the functions of the program complex in cases of its expansion at the request of customers. These libraries serve as a kind of multifunctional designer, which helps to qualitatively and efficiently expand the capabilities of the server and browser parts of the software package. In Table 1 shows a brief description of the functionality of the basic algorithms. The need to implement the possibilities of expanding the project is due to the fact that, as practice has shown, the original software specification is only the basis of the project and cannot always meet the growing demands of customers in the process of software using. As a result, the addition and creation of new system functionality is required. The structural diagram of the developed algorithms is presented in Figure 2.

Table 1. Brief description of the functionality of the basic algorithms

\begin{tabular}{|l|l|}
\hline \multicolumn{1}{|c|}{ Module Name } & \multicolumn{1}{|c|}{ Algorithm Assignment } \\
\hline greenlib.graphics.draw & $\begin{array}{l}\text { Common classes and interfaces for graphics output. } \\
\text { Provide the ability to modify cross-platform user interface. }\end{array}$ \\
\hline greenlib.graphics.draw.android & Platform-specific binding to the Android ${ }^{\mathbb{B}}$ operating system \\
\hline greenlib.graphics.draw.swing & Linking to Java ${ }^{\mathbb{B}}$ OpenGL \\
\hline greenlib.graphics.input & $\begin{array}{l}\text { System for recursive handling of mouse or touch screen } \\
\text { events (search for components and event delivery to a } \\
\text { component) }\end{array}$ \\
\hline greenlib.graphics.input.android & Linking greenlib.graphics.input to standard Android ${ }^{\mathbb{B}}$ input \\
\hline greenlib.graphics.input.swing & Linking greenlib.graphics.input to standard input \\
\hline greenlib.io & $\begin{array}{l}\text { Improved serialization system (easy to use and faster than } \\
\text { standard) }\end{array}$ \\
\hline greenlib.io.network & $\begin{array}{l}\text { The system of client-server transmission of byte arrays or } \\
\text { buffers over the network. Additionally, a system of } \\
\text { extensions is implemented for archiving, encrypting or } \\
\text { checking data for integrity. }\end{array}$ \\
\hline greenlib.io.network.protocol & $\begin{array}{l}\text { Allows to build on top of a simple protocol implemented in } \\
\text { greenlib.io.network }\end{array}$ \\
\hline greenlib.io.network.protocol.format & $\begin{array}{l}\text { Implementing the protocol } \\
\text { greenlib.io.network.protocol and serializing greenlib.io }\end{array}$ \\
\hline greenlib.io.network.protocol.json & $\begin{array}{l}\text { Implementing the protocol } \\
\text { greenlib.io.network.protocol and serializing in Json }\end{array}$ \\
\hline Monitoring system and console for servers \\
\hline greenlib.administrator & Supporting code to generate source code \\
\hline greenlib.physics & $\begin{array}{l}\text { Mathematical library for working with vectors, matrices } \\
\text { and objects }\end{array}$ \\
\hline
\end{tabular}

In the graphic visualization, two Android $^{\circledR}$ packages (an operating system for smartphones, etc.) for mobile phones and tablets, a swing (library for creating a graphical interface in $\mathrm{Java}^{\circledR}$ ) - for computers are used. The draw library includes interfaces and functions for drawing on tablets and mobile devices. Drawing is done with the help of triangles and their fill textures. Graphical visualization uses only the browser. The server stores the data, accepts requests and issues responses, and graphical drawing is performed by the browser. Respectively, three graphic libraries and three libraries for entering events from the keyboard and mouse are located in greenlib.graphics and belong to the browser part of the project.

Libraries greenlib.io ... (I/O) are designed to transfer data over the network and store data on the server, greenlib.administrator - to assist in debugging and monitoring the server. Using the greenlib.generator library, part of the greenlib.io code was generated, which reduced the number of potential errors and accelerated development.

The auxiliary library greenlib.physics was used for mathematical calculations in greenlib.graphics.draw. All libraries are written in Java ${ }^{\circledR}$, as an example is presented part of 
the developed mathematical module greenlib.physics.

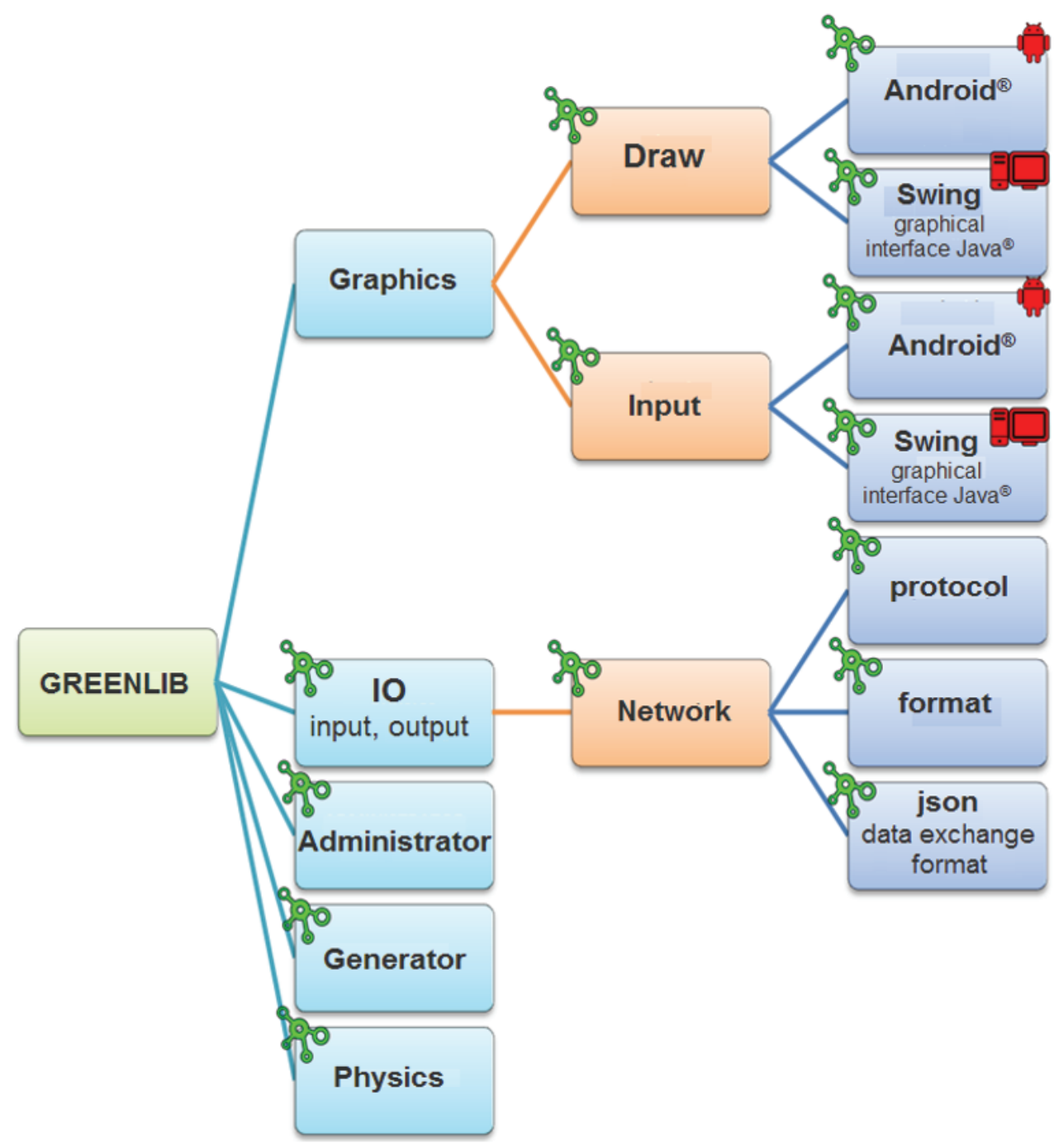

Fig. 2. Library hierarchy for the information subsystem of personnel management: (functionally complete program fragment); 를 - platform-specific code for PC (Windows ${ }^{\circledR}$, Linux ${ }^{\circledR}$ ); - platform-specific code for Android ${ }^{\mathbb{B}}$ (tablets, phones, smart-TVs).

The subsystem for assessing the safety level of mining operations by geomechanical factors evaluates the parameters geomechanical monitoring of the "support-rocks" system, it is proposed to combine factors of expected technical risks for the system to lose its stability by way of integrating the probability estimates of informative parameters of the rock mass and roadway state. Two integrated indices of safety were formed for each group of parameters consisting of $m$ indicators (standardized or normalized in the range from 0 to 1$)$.

The first indicator $R_{\max }$ reflects level of maximum technical risk and is determined by the value or rate of change of the monitoring parameter, which characterizes the most unacceptable state of the control object by choosing a maximum value from the calculated functions of the risk value distribution: 


$$
R_{\max }=\left\{\begin{array}{l}
R\left(E_{r}\right)=k r_{1} R\left(E_{r}^{1}\right)+k r_{2} R\left(E_{r}^{2}\right)+\ldots+k r_{m_{1}} R\left(E_{r}^{m_{1}}\right) \\
R\left(E_{s}\right)=k s_{1} R\left(E_{s}^{1}\right)+k s_{2} R\left(E_{s}^{2}\right)+\ldots+k s_{m_{2}} R\left(E_{s}^{m_{2}}\right) \\
R\left(E_{g}\right)=k g_{1} R\left(E_{g}^{1}\right)+k g_{2} R\left(E_{g}^{2}\right)+\ldots+k g_{m_{3}} R\left(E_{g}^{m_{3}}\right) \\
R\left(K_{r}\right)=k r_{1} R\left(K_{r}^{1}\right)+k r_{2} R\left(K_{r}^{2}\right)+\ldots+k r_{m_{4}} R\left(K_{r}^{m_{4}}\right) \\
R\left(K_{g}\right)=k g_{1} R\left(K_{g}^{1}\right)+k g_{2} R\left(K_{g}^{2}\right)+\ldots+k g_{m_{5}} R\left(K_{g}^{m_{5}}\right)
\end{array}\right.
$$

where $m_{1}, m_{2}, m_{3}$ are the number of risk factors, which affect stability of the roof $\left(E_{r}\right.$ factor), walls ( $E_{s}$ factor) and floor ( $E_{g}$ factor) of the roadways, respectively; $m_{4}, m_{5}$ are the number of risk factors, which can cause sudden failure of the roof ( $K_{r}$ factor) and sudden raising of the floor $\left(K_{g}\right.$ factor); $R\left(E_{r}\right), R\left(E_{s}\right), R\left(E_{g}\right), R\left(K_{r}\right), R\left(K_{g}\right)$ are potential risk factors of emergency caused by the factors of $E$ type (volume of inelastic deformation zones, displacement of roadway contour, etc.) and $K$ type (changed displacement rate, stress, volume of zones with continuity break, etc.); $K_{r}, K_{s}, K_{g}$ are normalized weighting factors, which can cause potential risk for specific factors in the group.

The second indicator $R_{\Sigma}$ reflects an integrated technical risk of the "support-rocks" system destruction, which is determined by sum of all of the risks:

$$
R_{\Sigma}=k_{1}^{v} \sum_{i=1}^{m_{1}} R\left(E_{r}\right)_{i}+k_{2}^{v} \sum_{i=1}^{m_{2}} R\left(E_{s}\right)_{i}+k_{3}^{v} \sum_{i=1}^{m_{3}} R\left(E_{g}\right)+k_{4}^{v} \sum_{i=1}^{m_{4}} R\left(K_{r}\right)_{i}+k_{5}^{v} \sum_{i=1}^{m_{5}} R\left(K_{g}\right)_{i}
$$

where $k_{1}{ }^{v}, k_{2}{ }^{v}, k_{3}{ }^{v}, k_{4}{ }^{v}, k_{5}{ }^{v}$ are normalized weighting factors of influence of each factor group, $k_{1}^{v}+k_{2}^{v}+k_{3}^{v}+k_{4}^{v}+k_{5}^{v}=1$.

The first indicator is used by the ISS to control entering of the control object to the emergency mode and to determine a factor, which requires urgent interruption, and the second indicator is used for the total assessment of the object current state.

To validate the risk criteria used by the ISS for assessing the job safety with taking into account geomechanical factors, typical scenarios of distribution of zones with inelastic deformation and stress changes in the rock mass are identified (an example is shown in Figure 3). With the help of method of mathematical modeling, the geomechanical situational models were calculated, which assumed use of different types of supports, increase of load on the "support-rocks" system, and changing conditions of the rocks bedding and water intrusion.

Algorithms were tested on example of typical organizational structure of the mines and approved in the production conditions. The results showed that the ISS improved rate of the underground mining jobs safety through: the integration of geomechanical data obtained from various measurements and information sources into one information field; support of decision-making process at various levels and provision of up-to-date information; organizational adaptation to the management structures; more detailed data processing on the base of mathematical models; criteria assessing rate of danger for the control object by methods fuzzy logics; personnel interaction via stationary and mobile interfaces.

"The Methodical Recommendations on How to Use the Information System for Ensuring the Underground Mining Jobs Safety on the Basis of Geomechanical State of the Rock Mass" were developed including the ISS functioning, preparation and deployment and specificity of its application in different mining enterprises. The Methodical Recommendations were successfully tested and implemented in the Institute of Safety and Ecology in the Mining and Metallurgical Industry, SHES "Kryvyi Rih National University" of Ministry of Education and Science of Ukraine and by other mining companies. 

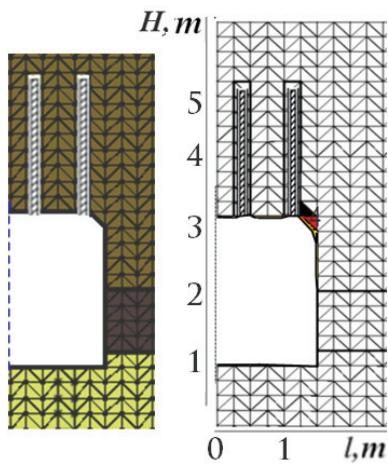

a)

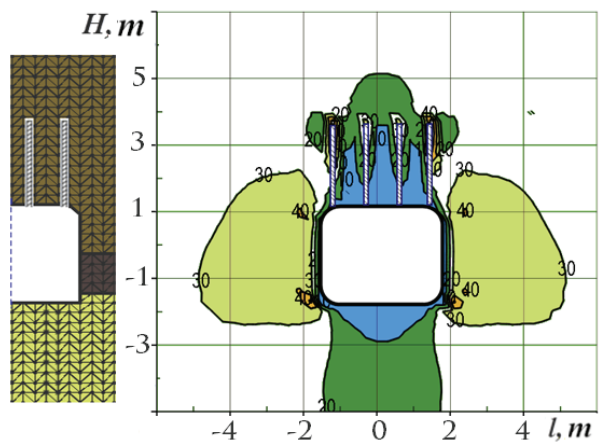

f)

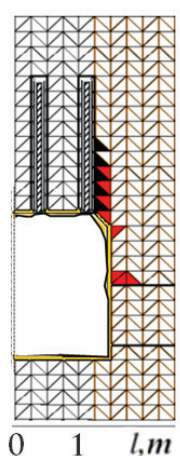

b)

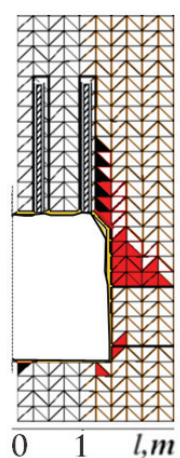

c)

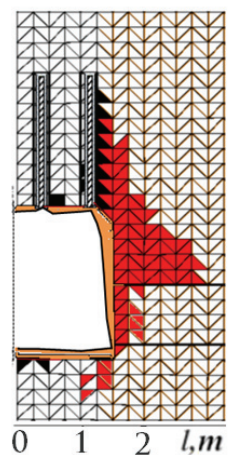

d)

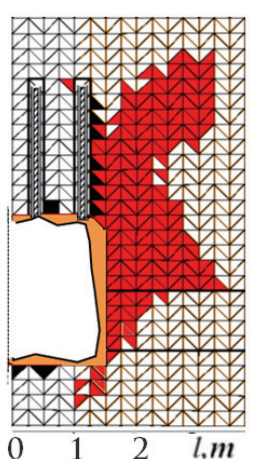

e)

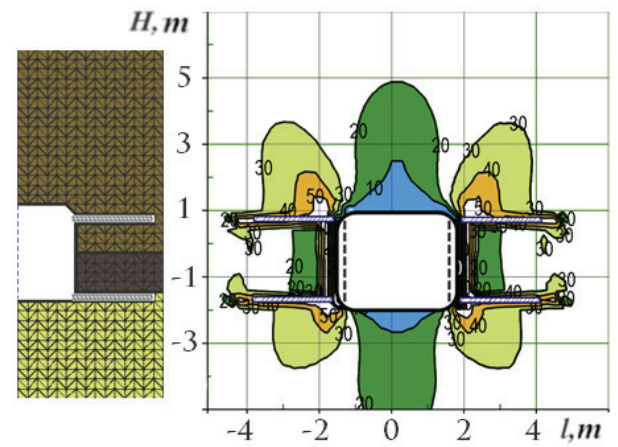

g)

Levels of maximum principal stresses, $\mathrm{MPa}(\mathrm{f}, \mathrm{g})$

$$
\begin{array}{lllll}
<10 & 10-20 & 20-30 & 30-40 & 40-50 \quad>50
\end{array}
$$

Fig. 3. Scenarios of inelastic deformation zone behavior in the rock massif around the roadway supported by the roof bolting at increasing depth of the mining jobs $(\mathrm{a}-250 \mathrm{~m}, \mathrm{~b}-500 \mathrm{~m} ; \mathrm{c}-750 \mathrm{~m}$; d - $1250 \mathrm{~m}$; e $-1500 \mathrm{~m}$ ) and changes of maximal stresses at different methods of the roof supporting (f, g); $\square$ - zones with destruction caused by shifting forces (a-e); $\square$ - zones with the continuity break (a-e).

\section{Conclusions}

1. Totally up to half of all accidents and accidents are caused by geomechanical factors, which occurring the form of uncontrolled deformation of the rock mass, destruction of the roof supports and blockage of the roadways. The second reason is the human factor. One of the ways to reduce number of accidents and injuries is to ensure more strict labor discipline of employees and to prevent scenarios of negative geomechanical processes with the help of the state-of-the art information systems.

2. Two integral indicators of safety are formed. The first indicator determines the maximum level of technical risk and is defined by maximal values selected from the functions of the groups related to the risk of losing stability of the roadway roof, walls and floor. The second indicator reflects an integrated geotechnical risk for the system to lose its stability and is determined by the values and rate of monitoring parameters changing. The first indicator is used to control entering of the control object to the emergency mode and to determine a factor, which requires urgent interruption, and the second indicator is used for the total assessment of the object current state. 
3. The information safety system architecture and some basic algorithms were developed. The system differs by its methods for prompt predicting and assessing of different scenarios of geomechanical process development, and which includes the following subsystems: a basic client-server subsystem with functions of interaction between the personnel and management of the enterprise; a reference and information subsystem, which supports a decision making process, accumulates data and analyzes technical documentation; a subsystem for analyzing the job safety by geomechanical factors and for assessing of the "support-rocks" system state basing on the risk criteria and mathematical fuzzy logics.

4. "The Methodical Recommendations on How to Use the Information System for Ensuring the Underground Mining Jobs Safety on the Basis of Geomechanical State of the Rock Massif" were developed including the ISS functioning, preparation and deployment and specificity of its application in different mining enterprises.

\section{References}

1. M Jiaqi, D. Hong, Safety Science 93 (2017)

2. S. Gerald, R.M. Hill, L. Jefferey, Journal of Safety Research 44 (2013)

3. N. Baisheng, H. Xin, S. Xin, L. Anjin, Safety Science 88 (2016)

4. Shevchenko, V.G. (2016). Developing methods for increasing readiness of the managers of coal mine divisions to accident-free operation according to quantitative estimations of theirpersonality characteristics, Scientific bulletin of National Mining University. 6. 114-119

5. Shevchenko, V.G. (2017). Research on the influence of miners' energy expenditure on coal mining efficiency, Scientific bulletin of National mining university, 3, 140-146

6. Ikonnikova, N.A., Korsun, V.I., Slaschev, A.I., Yalansky, Alex. A., Yalansky, A.A. (2015). Modelirovanie i kontrol dinamicheskih protsessov $\mathrm{V}$ zadachah otsenki sostoyaniya geotehnicheskih system. Dnipro: National mining university

7. Slashchev, A.I. (2016). Justification of the parameters of the information system assuring the underground mining safety, Scientific bulletin of National mining university, 1. 77-85

8. Shevchenko, V.G., Slashchov, A.I. (2018). Informatsionnyie sistemy bezopasnosti i proizvoditelnosti podzemnyih gornyih rabot. Kyiv: Naukova Dumka 